\title{
Reality of the Wigner Functions and Quantization
}

\author{
Sadollah Nasiri ${ }^{1,2}$ and Samira Bahrami ${ }^{1}$ \\ ${ }^{1}$ Department of Physics, Zanjan University, P. O. Box 45196-313, Zanjan 45371-38111, Iran \\ ${ }^{2}$ Department of Physics, Institute for Advanced Studies in Basic Sciences (IASBS), P. O. Box 45195-1159, Zanjan 45195-1159, Iran
}

Correspondence should be addressed to Samira Bahrami, bahrami.samira@gmail.com

Received 11 January 2009; Accepted 8 May 2009

Recommended by Nicholas Bigelow

Here we use the extended phase space formulation of quantum statistical mechanics proposed in an earlier work to define an extended lagrangian for Wigner's functions (WFs). The extended action defined by this lagrangian is a function of ordinary phase space variables. The reality condition of WFs is employed to quantize the extended action. The energy quantization is obtained as a direct consequence of the quantized action. The technique is applied to find the energy states of harmonic oscillator, particle in the box, and hydrogen atom as the illustrative examples.

Copyright ( $) 2009$ S. Nasiri and S. Bahrami. This is an open access article distributed under the Creative Commons Attribution License, which permits unrestricted use, distribution, and reproduction in any medium, provided the original work is properly cited.

\section{Introduction}

Among different methods proposed for the quantum statistical mechanics, one may employ the phase space distribution functions to calculate the average quantities. Wigner [1] pioneered in this direction. Based on the quantum state functions he proposed distribution functions with interesting properties and wide range of applicability. Since then many authors have examined Wigner's distribution functions, and an amazingly large amount of literature has been amassed. Wigner himself used them to make quantum corrections for thermodynamic equilibrium. Moyal [2] and Husimi [3] developed Wigner's technique extensively. They also showed that averaging by WFs is equivalent, in quantum mechanics, by an averaging in which noncommutating functions of operators $\hat{p}$ and $\hat{q}$ are ordered according to Weyl's ordering rule [4]. Further improvements were made by Margenau and Hill [5], Glauber [6], Mehta [7], O'Connell and Wigner [8], Hillery et al. [9], and Agarwal [10]. Another way of studying the quantum statistical mechanics is proposed by Sobouti and Nasiri $[11,12]$ by generalizing the principle of least action in configuration space to one in phase space. In this method the phase space coordinates are treated as independent variables on the virtual system point trajectories in phase space. This further permits a definition of extended phase space (EPS), extended lagrangians and hamiltonians. A subsequent canonical quantization procedure or using the path integral technique [13] through these extended entities gives a formulation for quantum statistical mechanics. To demonstrate the simplicity and advantages of the EPS formulation, the technique is applied to solve a few problems. Treatment of Bloch's theory, partition functions for simple harmonic, and linear potentials are worked out by Nasiri et al. [12]. Obtaining a unique expression for conductivity of a dissipative system of charged particles in different gauges is done using EPS technique by Khademi and Nasiri [14]. Considerable assistance of the EPS technique for studying the dissipative quantum systems is shown by Nasiri and Safari [15] and Razavy [16]. Here we use the later method to obtain an extended hamiltonian governing the evolution of WFs by an appropriate canonical transformation in EPS. An arbitrary extended lagrangian is then obtained by Legender's transformation. The extended action obtained by this lagrangian is quantized upon the reality condition of WFs that directly yields the quantization of energy. The layout of the paper is as follows. In Section 2, a brief review of the EPS formalism is introduced. In Section 3 the extended hamiltonian and lagrangian are introduced for WFs, and the quantization of extended action and energy are illustrated by a few examples. Section 4 is devoted to conclusions. 


\section{Background}

In this paper we use a direct approach to quantum statistical mechanics that are proposed by extending the conventional phase space and by applying the canonical quantization procedure to the extended quantities in this space (the EPS formalism).

2.1. EPS Formalism. In this formalism an extended lagrangian is introduced as follows [11]:

$$
\mathcal{L}(p, q, \dot{p}, \dot{q})=-\dot{p} q-\dot{q} p+L^{p}(p, \dot{p})+L^{q}(q, \dot{q}),
$$

where $L^{p}(p, \dot{p})$ and $L^{q}(q, \dot{q})$ are lagrangians in $\mathbf{p}$ and $\mathbf{q}$ space, and $p=\left\{p_{i}\right\}, q=\left\{q_{i}\right\}$ are the collection of generalized momenta and coordinates specifying a system, respectively. Assuming the phase space coordinates $\mathbf{p}$ and $\mathbf{q}$ to be independent variables on the virtual trajectories allows one to define momenta $\pi_{p}$ and $\pi_{q}$, conjugate to $\mathbf{p}$ and $\mathbf{q}$, as follows:

$$
\begin{aligned}
& \pi_{p}=\frac{\partial \mathcal{L}}{\partial \dot{p}}=\frac{\partial L^{p}}{\partial \dot{p}}-q, \\
& \pi_{q}=\frac{\partial \mathcal{L}}{\partial \dot{q}}=\frac{\partial L^{q}}{\partial \dot{q}}-p .
\end{aligned}
$$

Then, using these extended momenta, one may define an extended hamiltonian as follows:

$$
\begin{aligned}
\mathscr{H}\left(p, q, \pi_{p}, \pi_{q}\right) & =\pi_{p} \dot{p}+\pi_{q} \dot{q}-\mathcal{L}(p, q, \dot{p}, \dot{q}) \\
& =H\left(p+\pi_{q}, q\right)-H\left(p, q+\pi_{p}\right) .
\end{aligned}
$$

Using the quantization rule [11], one gets the following dynamical equation:

$$
\begin{aligned}
i \hbar \frac{\partial \chi}{\partial t} & =\mathcal{H} \chi \\
& =\left\{H\left(p-i \hbar \frac{\partial}{\partial q}, q\right)-H\left(p, q-i \hbar \frac{\partial}{\partial p}\right)\right\} \chi \\
& =\sum_{n=1} \frac{(i \hbar)^{n}}{n !}\left\{\frac{\partial^{n} H}{\partial p^{n}} \frac{\partial^{n}}{\partial q^{n}}-\frac{\partial^{n} H}{\partial q^{n}} \frac{\partial^{n}}{\partial p^{n}}\right\} \chi,
\end{aligned}
$$

where $\chi(p, q, t)=\psi(q, t) \varphi^{*}(p, t) e^{-(i p q / \hbar)}$ is distribution function in EPS where $\psi(q, t)=(1 / \sqrt{2 \pi \hbar}) \int d p \varphi(p, t) e^{i p q / \hbar}$, $\pi_{p}=-i \hbar(\partial / \partial p)$, and $\pi_{q}=-i \hbar(\partial / \partial q)$. The averaging rule for an observable $O(p, q)$, a c-number operator in this formalism specified by antistandard ordering rule, is

$$
\langle O(p, q)\rangle=\int O(p, q) \chi^{*}(p, q) d p d q .
$$

For more details, one may consult Hillery et al. [9] and Sobouti and Nasiri [11].

2.2. The Extended Canonical Transformation and WFs. The quantization procedure of Section 2 assumes $\mathbf{p}$ and $\mathbf{q}$ as c-numbers, similar to Hamilton's formulation of classical mechanics. Therefore, the rules of linear canonical transformations in classical mechanics are directly applicable to EPS quantities. These linear canonical transformations correspond to the unitary transformations in quantum mechanics [17].

A simple infinitesimal extended canonical transformation on $p, q, \pi_{p}$, and $\pi_{q}$ gives WFs and the corresponding evolution equation. Let

$$
\begin{array}{ll}
p \longrightarrow p+\delta \lambda \pi_{q}, & \pi_{p} \longrightarrow \pi_{p}, \\
q \longrightarrow q+\delta \lambda \pi_{p}, & \pi_{q} \longrightarrow \pi_{q},
\end{array}
$$

where $\delta \lambda \ll 1$, the generator of this infinitesimal transformation is

$$
G=\pi_{p} \pi_{q} .
$$

The corresponding unitary transformation for finite $\lambda$ is

$$
U=e^{i \lambda G / \hbar}=e^{i \lambda \hbar \partial^{2} / \partial p \partial q}, \quad U U^{+}=1 .
$$

The above transformation, for $\lambda=-1 / 2$, transforms the distribution $\chi(p, q, t)$, to those of the Wigner [1]. That is,

$$
\begin{aligned}
W(p, q, t) & =U \chi(p, q, t) \\
& =\int \psi\left(q+\frac{1}{2} \hbar \tau\right) \psi^{*}\left(q-\frac{1}{2} \hbar \tau\right) e^{i p \tau} d \tau,
\end{aligned}
$$

and the evolution equation (4) changes into the Wigner equation:

$$
\begin{aligned}
\frac{\partial W}{\partial t}= & -p \frac{\partial W}{\partial q} \\
& +\sum_{n=0} \frac{1}{(2 n+1) !}\left(\frac{\hbar}{2 i}\right)^{2 n} \frac{\partial^{2 n+1} V}{\partial q^{2 n+1}} \frac{\partial^{2 n+1} W}{\partial p^{2 n+1}} .
\end{aligned}
$$

A similar technique could be used to obtain other distribution functions and their corresponding ordering rules [1820].

\section{The Extended Hamiltonian and Extended Lagrangian for WFs}

By transformation (8) the extended hamiltonian of (3) becomes

$$
\begin{aligned}
& \mathscr{H}_{w}\left(p, q, \pi_{p}, \pi_{q}\right) \\
& \quad=H\left(p+\frac{1}{2} \pi_{q}, q-\frac{1}{2} \pi_{p}\right)-H\left(p-\frac{1}{2} \pi_{q}, q+\frac{1}{2} \pi_{p}\right) .
\end{aligned}
$$

The subscript $w$ denotes the quantities for WFs. For $H=$ $p^{2} / 2 m+V(q)(11)$ gives

$$
\begin{aligned}
\mathscr{H}_{w}= & \frac{1}{2 m}\left(p+\frac{1}{2} \pi_{q}\right)^{2}+V\left(q-\frac{1}{2} \pi_{p}\right) \\
& -\frac{1}{2 m}\left(p-\frac{1}{2} \pi_{q}\right)^{2}-V\left(q+\frac{1}{2} \pi_{p}\right) .
\end{aligned}
$$


The extended lagrangian, $\mathcal{L}_{w}$, for WFs may be defined by the following Legendre transformation:

$$
\begin{aligned}
\mathscr{H}_{w}\left(p, q, \frac{\partial \mathcal{L}_{w}}{\partial \dot{p}}, \frac{\partial \mathcal{L}_{w}}{\partial \dot{q}}\right) \\
=\dot{p} \frac{\partial \mathcal{L}_{w}}{\partial \dot{p}}+\dot{q} \frac{\partial \mathcal{L}_{w}}{\partial \dot{q}}-\mathcal{L}_{w}(p, q, \dot{p}, \dot{q}) .
\end{aligned}
$$

Equation (13) serves as a differential equation to determine $\mathcal{L}_{w}$. Note that the extended lagrangians are not unique and they just serve to obtain the correct equations of motion through the Euler-Lagrange equations for configuration and momentum spaces.

The solutions of the Schrödinger equation can be written as [21]

$$
\psi(q, t)=R^{q}(q, t) e^{i S^{q}(q) / \hbar},
$$

where $S^{q}(q)$ is the ordinary action in $\mathbf{q}$ space defined by

$$
S^{q}(q)=\int L^{q}(q, \dot{q}, t) d t .
$$

By the same manner one may do in $\mathbf{p}$ space:

$$
\varphi(p, t)=R^{p}(p, t) e^{-\left(i S^{p}(p) / \hbar\right)},
$$

where

$$
S^{p}(p)=\int L^{p}(p, \dot{p}, t) d t
$$

is the action in $\mathbf{p}$ space. Substituting for $\psi$ and $\varphi$, from (14) and (16) in the expression given for $\chi$, one gets

$$
\chi=\psi \varphi^{*} e^{-(i p q / \hbar)}=R^{q} R^{p} e^{i\left(S^{q}+S^{p}-p q\right) / \hbar} .
$$

Now, let the extended action be defined as

$$
S_{\chi}(p, q)=\int \mathcal{L}(p, q, \dot{p}, \dot{q}, t) d t .
$$

Using (1), (15), and (17) one gets

$$
S_{\chi}(p, q)=S^{q}+S^{p}-p q,
$$

which is the same as appeared in the phase of (18). Hence,

$$
\chi=R_{\chi} e^{i S_{\chi}(p, q) / \hbar},
$$

where $R_{\chi}(p, q, t)=R^{p}(p, t) R^{q}(q, t)$. Therefore, in general, any phase space distribution function, $\rho(p, q, t)$ can be written in the form of an amplitude multiplied by a phase factor [13] as follows:

$$
\rho(p, q, t)=R_{\rho}(p, q, t) e^{i S_{\rho}(p, q) / \hbar},
$$

where $R_{\rho}(p, q, t)$ is the amplitude, and $S_{\rho}(p, q)$ is the corresponding extended action of $\rho(p, q, t)$. Both of $R_{\rho}$ and $S_{\rho}$ are real functions. In the same manner for WFs, as a possible distribution functions in the phase space, we formally have

$$
W(p, q, t)=R_{w}(p, q, t) e^{i S_{w}(p, q) / \hbar},
$$

where

$$
S_{w}(p, q)=\int \mathcal{L}_{w}(p, q, \dot{p}, \dot{q}, t) d t,
$$

and $\mathcal{L}_{w}$ is given by (13). However, it is well known that the WFs are real functions of the phase space variables. Thus, the phase $S_{w}(p, q)$ in (23) must satisfy the following constraint:

$$
\sin \left\{\frac{S_{w}(p, q)}{\hbar}\right\}=0
$$

or

$$
S_{w}(p, q)=n \pi \hbar, \quad n=0,1,2, \ldots .
$$

Equation (26) shows the quantization of extended action. As will be shown later, due to the uncertainty principle, the values of $n$ must be correctly chosen for a given problem. Note that the extended action in EPS is a function of phase space variables and is basically different from ordinary action $S^{q}(q)$ defined in configuration space. As an interesting result, one has $\cos \left\{S_{w}(p, q) / \hbar\right\}=\cos (n \pi)=(-1)^{n}$. Thus, the well known negativity of WFs, $w(p, q, t)=(-1)^{n} R_{w}(p, q, t)$, seems to have a quantum origin [22].

3.1. Energy Quantization. In this section we show that the quantization of the extended action of (26) is directly related to the quantization of energy. To see this we consider a few examples.

3.1.1. Harmonic Oscillator. For harmonic potential, $H=$ $p^{2} / 2 m+(1 / 2) m \omega^{2} q^{2}(12)$ gives

$$
\mathscr{H}_{w}\left(p, q, \pi_{p}, \pi_{q}\right)=\frac{p}{m} \pi_{q}-m \omega^{2} q \pi_{p} .
$$

By (13) one gets

$$
\frac{p}{m} \frac{\partial \mathcal{L}_{w}}{\partial \dot{q}}-m \omega^{2} q \frac{\partial \mathcal{L}_{w}}{\partial \dot{p}}=\dot{p} \frac{\partial \mathcal{L}_{w}}{\partial \dot{p}}+\dot{q} \frac{\partial \mathcal{L}_{w}}{\partial \dot{q}}-\mathcal{L}_{w}(p, q, \dot{p}, \dot{q}) .
$$

The extended lagrangian as the solution of differential equation (28) may be obtained as

$$
\mathcal{L}_{w}(p, q, \dot{p}, \dot{q})=\frac{\pi}{\omega}\left(\frac{p}{m} \dot{p}+m \omega^{2} q \dot{q}\right) .
$$

It can be shown that $\mathcal{L}_{w}$ satisfies the Euler-Lagrange equations in both configuration and momentum space. Using (24) and (29) one obtains

$$
\begin{aligned}
S_{w}(p, q) & =\frac{\pi}{\omega}\left(\frac{p^{2}}{2 m}+\frac{1}{2} m \omega^{2} q^{2}\right) \\
& =\frac{\pi}{\omega}\left(\mathrm{H}-\frac{1}{2} \hbar \omega\right),
\end{aligned}
$$

where $\mathrm{H}$ is the quantum mechanical Hamiltonian assuming the uncertainty principle, implicitly [23]. Equations (26) and (30) give

$$
E_{n}(p, q)=\left(n+\frac{1}{2}\right) \hbar \omega, \quad n=0,1,2,3, \ldots,
$$

as the quantization of energy of a harmonic oscillator. 
3.1.2. Particle in the Box. For a particle in a one dimensional box of side $\mathbf{L}$ located at the right side of origin, one has

$$
V(q)= \begin{cases}\infty, & q<0 \\ 0, & 0<q<L \\ \infty, & q>L\end{cases}
$$

For $H=p^{2} / 2 m$ one gets the following from (12):

$$
\mathscr{H}_{w}\left(p, q, \pi_{p}, \pi_{q}\right)=\frac{p}{m} \pi_{q} .
$$

By legendre's transformation on the above extended hamiltonian, one gets for an arbitrary extended lagrangian;

$$
\mathcal{L}_{w}(p, q, \dot{p}, \dot{q})=L \dot{p}
$$

Using (24) and (34) the extended action becomes

$$
S_{w}(p, q)=L p=L(2 m H)^{1 / 2} .
$$

Then, by (26) and squaring (35) one gets

$$
E_{n}=\frac{n^{2} \pi^{2} \hbar^{2}}{2 m L^{2}}, \quad n=1,2, \ldots,
$$

where $n$ cannot assume zero due to the uncertainty principle. Equation (36) shows the energy levels of a particle in the box.

3.1.3. Hydrogen Atom. For the electron in the hydrogen-like atom, as a bound system with $\mathbf{Z}$ protons, the hamiltonian is $p_{r}^{2} / 2 m+l^{2} / 2 m r^{2}-\left(z e^{2} / r\right)[24]$. By (12) one gets

$$
\begin{aligned}
& \mathscr{H}_{w}\left(p_{r}, r, \pi_{p_{r}}, \pi_{r}\right) \\
&=\frac{p_{r}}{m} \pi_{r}+\frac{2 \ell^{2} r \pi_{p_{r}}+2 m Z e^{2} r^{2} \pi_{p_{r}}+\left(m z e^{2} / 2\right) \pi_{p_{r}}^{3}}{2 m\left(r-(1 / 2) \pi_{p_{r}}\right)^{2}\left(r+(1 / 2) \pi_{p_{r}}\right)^{2}} .
\end{aligned}
$$

A suitable extended lagrangian can be obtained by (25) to be

$$
\begin{array}{r}
\mathcal{L}_{w}\left(p_{r}, r, \dot{p}_{r}, \dot{r}\right)=\frac{m Z}{2 \sqrt{2}} \pi e^{2}\left(\frac{p_{r}}{m} \dot{p}_{r}-\frac{\ell^{2} \dot{r}}{m r^{3}}+\frac{e^{2} \dot{r}}{r^{2}}\right) \\
\times\left(\frac{p_{r}^{2}}{2 m}+\frac{\ell^{2}}{2 m r^{2}}-\frac{z e^{2}}{r}\right)^{-3 / 2} .
\end{array}
$$

The extended action becomes

$$
S_{w}\left(p_{r}, r\right)=\sqrt{\frac{m}{2}} \pi Z e^{2} H^{-1 / 2}
$$

Then, by (37) and squaring (39) one gets

$$
\left|E_{n}\right|=\frac{m Z^{2} e^{4}}{2 \hbar^{2} n^{2}}=\frac{Z^{2} e^{2}}{2 a_{0} n^{2}}, \quad n=1,2, \ldots,
$$

where $a_{0}$ is the Bohr radius, and (40) gives the energy levels of the hydrogen atom. The same as the previous case $n$ starts from 1 due to the uncertainty principle.

\section{Conclusion}

The EPS formulation of quantum statistical mechanics permits the definition of extended hamiltonians and extended lagrangians. A canonical quantization in EPS gives a dynamical equation for the phase space distribution functions. Wigner's distribution functions and their corresponding evolution equation can be obtained by a simple extended canonical transformation in EPS. The extended hamiltonian through Legendre's transformation provides a differential equation for the corresponding extended lagrangian. Having the extended lagrangian one may define the extended action as a function of ordinary phase space variables. Applying the reality condition of the WFs yields the quantization of the extended action which in turn gives the energy quantization. The energy levels of the harmonic oscillator, the particle in the box and the hydrogen atom are worked out as a few illustrative examples.

\section{References}

[1] E. P. Wigner, "On the quantum correction for thermodynamic equilibrium,” Physical Review, vol. 40, no. 5, pp. 749-759, 1932.

[2] J. E. Moyal, "Quantum mechanics as a statistical theory," Mathematical Proceedings of the Cambridge Philosophical Society, vol. 45, no. 1, pp. 99-124, 1949.

[3] K. Husimi, "Some formal properties of the density matrix," Proceedings of the Physico Mathematical Society of Japan, vol. 22, pp. 264-314, 1940.

[4] H. Weyl, "Quantenmechanik und gruppentheorie [The theory of groups and quantum mechanics]," Zeitschrift für Physik A, vol. 46, no. 1-2, pp. 1-46, 1927.

[5] H. Margenau and R. N. Hill, "Correction between measurements in quantum theory," Progress of Theoretical Physics, vol. 26, no. 5, pp. 722-738, 1961.

[6] R. J. Glauber, "The quantum theory of optical coherence," Physical Review, vol. 130, no. 6, pp. 2529-2539, 1963.

[7] C. L. Mehta, "Phase-space formulation of the dynamics of canonical variables," Journal of Mathematical Physics, vol. 5, no. 5, pp. 677-686, 1964.

[8] R. F. O'Connell and E. P. Wigner, "Quantum-mechanical distribution functions: conditions for uniqueness," Physics Letters A, vol. 83, no. 4, pp. 145-148, 1981.

[9] M. Hillery, R. F. O’Connell, M. O. Scully, and E. P. Wigner, "Distribution functions in physics: fundamentals," Physics Reports, vol. 106, no. 3, pp. 121-167, 1984.

[10] G. S. Agarwal, "Wigner function description of quantum noise in interferometers," Journal of Modern Optics, vol. 34, no. 6-7, pp. 909-921, 1987.

[11] Y. Sobouti and S. Nasiri, "A phase space formulation of quantum state functions," International Journal of Modern Physics B, vol. 7, no. 18, pp. 3255-3272, 1993.

[12] S. Nasiri, Y. Sobouti, and F. Taati, "Phase space quantum mechanics-direct," Journal of Mathematical Physics, vol. 47, no. 9, Article ID 092106, 15 pages, 2006.

[13] S. Nasiri, "A path integral formulation of quantum state functions," Iranian Journal of Science \& Technology, vol. 19, pp. 183-190, 1995.

[14] S. Khademi and S. Nasiri, "Gauge invariant quantization of dissipative systems of charged particles in extended phase 
space," Iranian Journal of Science and Technology, vol. 26, no. 1, pp. 171-179, 2002.

[15] S. Nasiri and H. Safari, "A symmetric treatment of damped harmonic oscillator in extended phase space," Proceedings of the Institute of Statistical Mathematics, vol. 43, pp. 645-651, 2002.

[16] M. Razavy, Classical and Quantum Dissipative, Imperial Collage Press, London, UK, 2005.

[17] E. Merzbacher, Quantum Mechanics, John Wiley \& Sons, New York, NY, USA, 2nd edition, 1970.

[18] S. Nasiri, S. Khademi, S. Bahrami, and F. Taati, "A generalized rule for non-commuting operators in extended phase space," in Proceedings of the 4th International Symposium on Quantum Theory and Symmetries (QTS '05), vol. 4, pp. 820-826, Varna, Bulgaria, August 2005.

[19] S. Nasiri, "Quantum potential and symmetries in extended phase space," Symmetry, Integrability and Geometry: Methods and Applications, vol. 2, pp. 62-74, 2006.

[20] S. Khademi and S. Nasiri, "Operator gage symmetry in QED," Symmetry, Integrability and Geometry: Methods and Applications, vol. 2, pp. 13-20, 2006.

[21] P. R. Holland, The Quantum Theory of Motion, Cambridge University Press, Cambridge, UK, 1993.

[22] A. Kenfack and K. Zyczkowski, "Negativity of the Wigner function as an indicator of non-classicality," Journal of Optics $B$, vol. 6, no. 10, pp. 396-404, 2004.

[23] J. J. Sakurai, Modern Quantum Mechanics, Pearson Education, New Delhi, India, 1993.

[24] H. Goldstein, C. Poole, and J. Safko, Classical Mechanics, Addison-Wesley, Reading, Mass, USA, 3rd edition, 2002. 

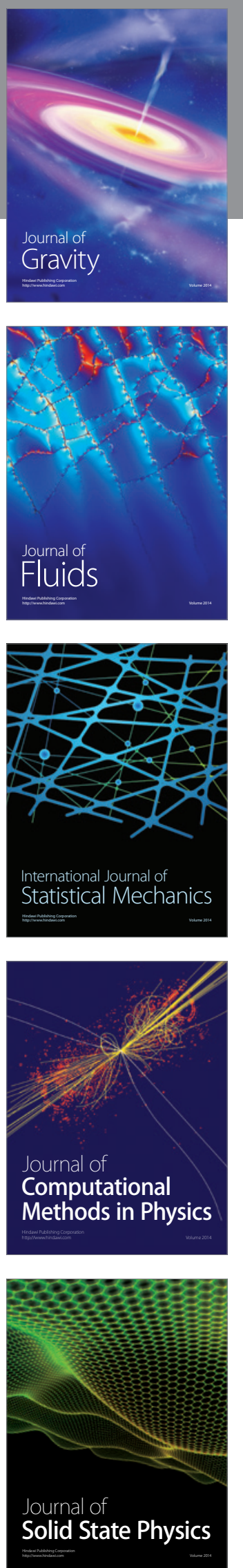

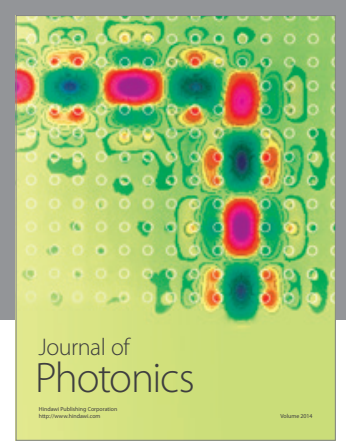

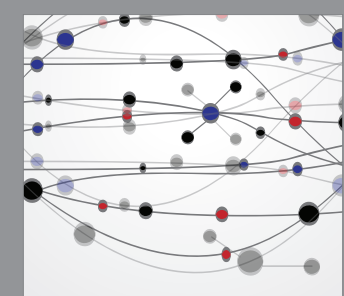

The Scientific World Journal
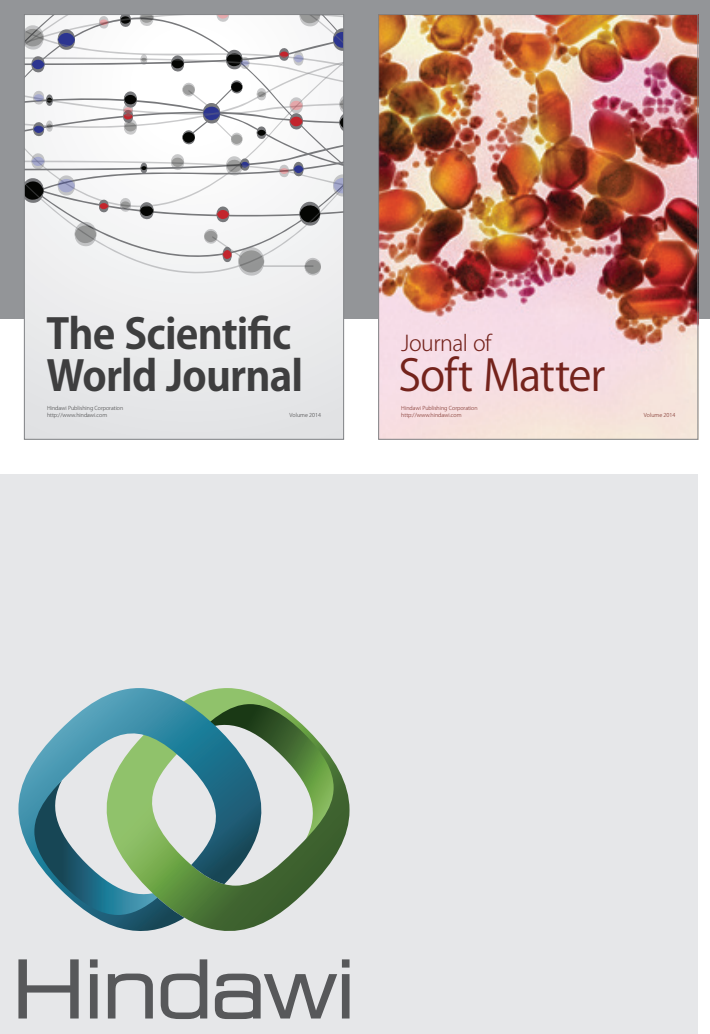

Submit your manuscripts at

http://www.hindawi.com
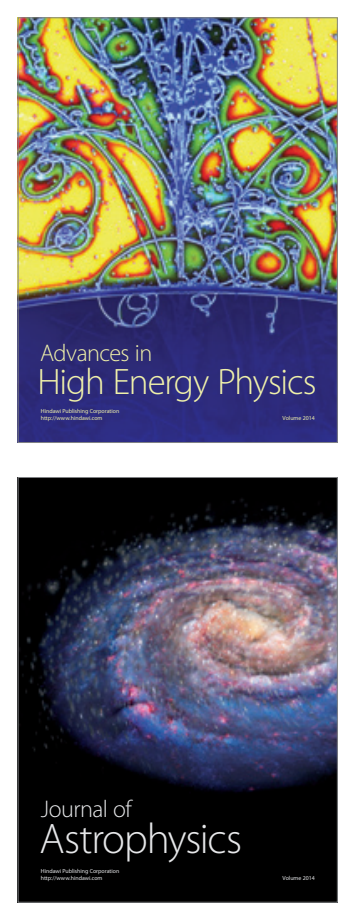
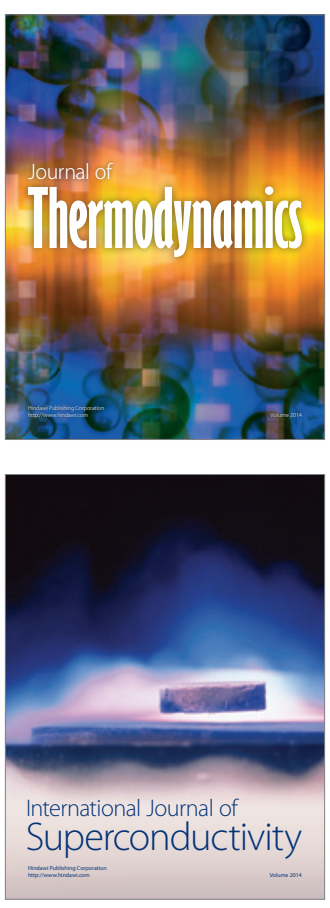
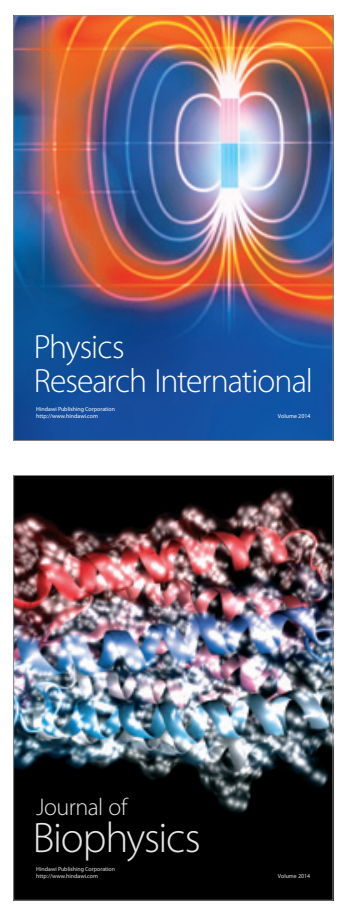
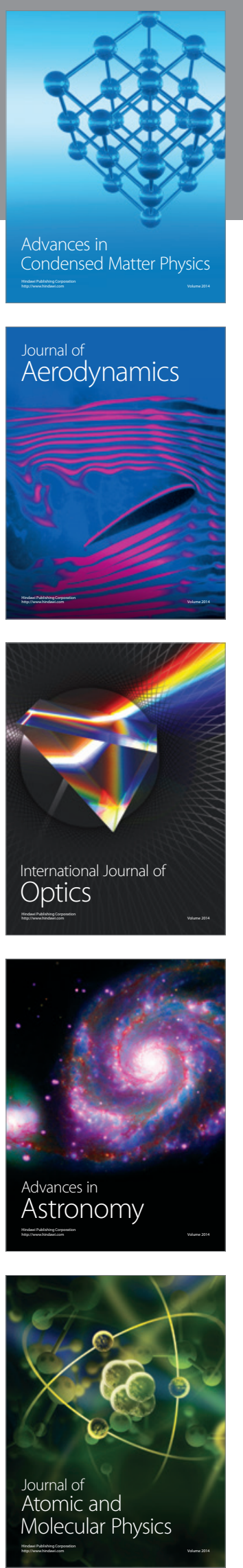